teachers reported more conduct disorders. Parents rated temperament to be more negative in emotionality and difficult-demanding. Teachers reported significantly lower academic achievement in PC group, especially in those with hyperactivity. Infants with PC also had increased incidence of sleeping or feeding problems. (Wolke D, Rizzo P, Woods S. Persistent infant crying and hyperactivity problems in middle childhood. Pediatrics June 2002;109:1054-1060). (Reprints: Dr Dieter Wolke, University of Hertfordshire, Department of Psychology, Wolke Research Group, Hatfield Campus, College Lane, Hatfield/Herts AL10 9AB, UK).

COMMENT. Infants with persistent crying and associated sleeping or feeding problems are at risk for hyperactive behavior and learning difficulties in childhood.

\title{
HEAD CIRCUMFERENCE IN AUTISM, ASPERGER SYNDROME AND ADHD
}

Occipitofrontal circumfcrence (OFC), measured at birth and after 16 months of age, was compared in 50 consecutive patients with Asperger syndrome, 50 diagnosed with autistic disorder, and 50 with ADHD and followed at the Department of Child and Adolescent Psychiatry, Goteborg University, Sweden. All three groups had mean OFCs at birth and after age 16 months that were larger than normal. Asperger patients' mean OFC was significantly greater than that of the autistic group and the ADHD group. Macrocephaly (defined as OFC $>2$ SDs and height and weight 1SD below that expected for the OCD) was present in 11 of 43 Asperger patients at birth and in 9 of 43 after 16 months. Corresponding rates were 4 of 42 in the autistic group, and 7 of 47 in the ADHD group at birth. Significant correlations between OFC at birth and after age 16 months occurred in the Asperger but not in the autistic or ADHD groups. OFC and IQ were not correlated. Two children with Asperger syndrome who had suffered perinatal asphyxia had microcephaly. Autistic spectrum disorders included a subgroup with macrocephaly and a relatively high level of functioning, with clinical presentation consistent with Asperger syndrome. (Gillberg C, de Souza L. Head circumference in autism, Asperger syndrome, and ADHD: a comparative study. Dev Med Child Neurol 2002;44:296-300). (Respond: Christopher Gillberg MD PhD, Department of Child and Adolescent Psychiatry, Goteborg University, Kungsgatan 12, SE-411 19, Goteborg, Sweden).

COMMENT. One in 4 children with Asperger syndrome and 1 in 10 of those with autistic disorder have macrocephaly when examined after 16 months of age. Among autistic children, macrocephaly is more typical of the highest functioning variant, Asperger syndrome, and is not characteristic of the moderate to low-functioning autistic spectrum disorder.

\section{TICS, ADHD AND PSYCHIATRIC COMORBIDITY}

The prevalence of teacher-rated tic behaviors in 3006 school children, from preschool to adolescence, was determined in a study of comorbid psychiatric symptoms at State University of New York, Stony Brook, NY. The prevalence of tic behaviors varied with age: preschoolers (22.3\%), elementary school children $(7.8 \%)$, and adolescents $(3,4 \%)$. Males were affected more than females, regardless of ADHD symptoms. Prevalence of tics was highest in the ADHD groups, but OCD and social phobia were more common in children with tics. (Gadow KD, Nolan EE, Sprafkin J, Schwartz J. Tics and psychiatric comorbidity in children and adolescents. Dev Med Child Neurol 2002;44:330-338). (Respond: Dr Gadow). 